MATHEMATICS OF COMPUTATION

Volume 67, Number 222, April 1998, Pages 619-639

S 0025-5718(98)00957-0

\title{
NUMERICAL CONFORMAL MAPPING BASED ON THE GENERALISED CONJUGATION OPERATOR
}

\author{
BAO CHENG LI AND STAVROS SYNGELLAKIS
}

\begin{abstract}
An iterative procedure for numerical conformal mapping is presented which imposes no restriction on the boundary complexity. The formulation involves two analytically equivalent boundary integral equations established by applying the conjugation operator to the real and the imaginary parts of an analytical function. The conventional approach is to use only one and ignore the other equation. However, the discrete version of the operator using the boundary element method (BEM) leads to two non-equivalent sets of linear equations forming an over-determined system. The generalised conjugation operator is introduced so that both sets of equations can be utilised and their least-square solution determined without any additional computational cost, a strategy largely responsible for the stability and efficiency of the proposed method. Numerical tests on various samples including problems with cracked domains suggest global convergence, although this cannot be proved theoretically. The computational efficiency appears significantly higher than that reported earlier by other investigators.
\end{abstract}

\section{INTRODUCTION}

For the last two decades or so the numerical generation of a boundary-fitted coordinate system (BFCS) has been attracting considerable attention among researchers within various branches of science and engineering. The aim of the technique is basically to construct curvilinear coordinate systems on physical 2-D or 3-D domains with a coordinate line or surface coinciding with the domain boundary. The philosophy behind this is that the problems under consideration may be re-cast in terms of the new coordinates and, hopefully, their solution may be more easily achieved. Equivalently, the technique can be regarded as a means of transforming an arbitrarily shaped physical domain into a simple canonical domain, such as a rectangle or a unit circle. The solution of the problem in the canonical domain, usually easier due to the simpler boundary conditions, is then transformed back to the original domain.

The most vigorous investigations on the subject have been undertaken principally within the field of fluid dynamics. This is largely due to the fact that most of the present computational fluid dynamic (CFD) problems are solved by finite difference (FD) techniques which, nevertheless, cannot be easily adapted to complicated boundary conditions. Thus the domain transformation can significantly

Received by the editor September 7, 1995 and, in revised form, September 19, 1996.

1991 Mathematics Subject Classification. Primary 30C30; Secondary 65 N38.

Key words and phrases. Numerical conformal mapping, conjugation operator, boundary integral equation. 
simplify the CFD coding. The numerical generation of BFCS has also proved to be useful for finite element (FE) grid generations.

There are basically three types of procedures for BFCS generation. The first uses algebraic transformations which are fast but usually do not produce sufficiently smooth coordinate systems. The second type constructs a BFCS from the solutions of partial differential equations (PDE's), which are either elliptic or hyperbolic. Chu [1] seems to be the first to adopt this method in FD solutions of 2-D fluid flow problems. Mastin and Thompson [2] extended this idea to 3-D problems. There have been numerous investigations on controlling the distribution of the coordinate lines, on generating orthogonal systems and on the trade-off between orthogonality and line spacing [3, 4]. A very comprehensive survey of the research prior to 1981, containing nearly four hundred references, has been presented by Thompson et al. [5]. More recently, Sparis [6] has proposed the use of biharmonic equations for BFCS generation. His method allows direct and complete control of the mesh point location on the boundary and the angle of intersection of the coordinate lines with the boundary.

The third technique is based on the numerical conformal mapping (NCM) which defines a curvilinear system locally resembling a Cartesian frame, and is generally preferable to other methods. Normally the PDE's are less distorted, and the Laplace equation in particular remains unchanged under conformal mapping. However, the construction of NCM usually requires the solution of a non-linear integral equation for which there are few generically effective algorithms. This is considered its main disadvantage.

The numerical conformal mapping technique can be used to transform a general domain into the interior of a unit circle. This effort is justified by the well-known fact that a Dirichlet or a Neumann problem for the Laplace equation can be solved explicitly by Poisson's or Dini's formula if the problem domain is the interior of a circle. For problems in solid mechanics and fracture mechanics, NCM can be combined with the boundary element method to produce highly efficient solutions. NCM is also a competitive method to generate FE and FD grids.

The technique is most often applied to fluid dynamics problems. For example, NCM has been employed for the calculation of transonic flow over airfoils [7]. Such a mapping greatly simplifies the boundary conditions and ensures that the non-linear PDE's in the computational plane are only slightly more complicated than in the physical plane. NCM has been found particularly useful in 2-D incompressible, irrotational free-surface flow problems $[8,9]$.

There are conventionally four methods in the constructive theory of conformal mapping: (1) a proper mapping for the problem under consideration may be fortuitously found by trying several transformations such as polynomial, rational or elliptic functions; (2) in the case of a nearly circular problem domain, there exists a closed form analytic function which approximately maps the domain onto the interior of a unit circle; (3) a general polygon can be mapped onto a circle or a half-plane by using the Schwarz-Christoffel formula; (4) with a domain bounded by circular arcs, the mapping can be constructed by solving a second order linear differential equation and determining the associated parameters. All four approaches have been thoroughly treated by Nehari [10]. It has to be emphasised that the classical methods, notwithstanding their elegance, are principally of academic rather than practical interest. This is so because the first two methods are only applicable 
to very special domains, while the application of the last two involves excessive difficulty in evaluating the associated parameters.

\section{NUMERICAL CONFORMAL MAPPING}

Since closed form solutions to conformal mapping are too limited to cope with general problems, modern developments on the subject are mainly directed towards their numerical construction. The methods of NCM fall generally into two classes: those which map the problem domain onto a canonical domain such as the interior of a unit circle, and those by which the mapping is performed in the reverse direction. Since an analytic function is entirely determined by its boundary value, the primary effort involved in NCM is to construct the boundary correspondence function (BCF) which establishes a bijective mapping between the boundaries of the problem and the canonical domains. The BCF is usually governed by a certain boundary integral equation (BIE) which can be numerically solved by discretizing the boundary at $n$ points and transforming the integral equation into an algebraic system.

In the methods developed by Symm [11, 12] and its variants [13, 14, 15], which belong to the first group, the BIE's are generally linear and their solution requires at least $n^{2} \log _{2} n$ operations. On the other hand, the integral equations associated with the methods in the second group are usually non-linear and they can be solved iteratively by successively applying the fast Fourier transform (FFT), hence the computation is $O\left(n \log _{2} n\right)$. There is a great variety of methods for mapping the unit circle onto the problem domain. They include the successive conjugation methods of Theodorsen [16, 17], Timman [18] and Friberg [19]; the projection method of Bergstrom [20] and the Newton methods of Vertgeim [21], Wegmann [22, 23, 24], Hübner [8] and Fornberg [25, 26]. The numerical computation of the SchwarzChristoffel transformation [27, 28, 29] also belongs to the second group, though, in this case, a system of transcendental equations for the accessory parameters needs to be solved rather than a BIE for the BCF.

The following discussion serves as an introduction to some key concepts focusing on three of the most original and efficient methods. For simplicity, only simplyconnected domains are considered. The problem of conformal mapping is formally stated below.

Problem A. Let $\Omega_{z}$ and $\Omega_{w}$ be simply-connected domains in complex $z$ - and $w$ planes bounded by $C_{z}$ and $C_{w}$, respectively. It is required to find an analytic function $f(z)$ which maps $\Omega_{z}$ onto $\Omega_{w}$ and assumes every value in $\Omega_{w}$ exactly once.

(i) Theodorsen's method. In Problem $\mathrm{A}, \Omega_{z}$ is the interior of a unit circle and $\Omega_{w}$ is star-like with respect to the origin. The boundary $C_{w}$ is given in polar coordinates,

$$
C_{w}: w(\varphi)=\rho(\varphi) e^{i \varphi}, \quad 0 \leq \varphi \leq 2 \pi .
$$

The boundary value of $f(z)$ can be written in terms of the boundary corresponding function $\varphi(\theta)$,

$$
f\left(e^{i \theta}\right)=\rho[\varphi(\theta)] e^{i \varphi(\theta)}, \quad 0<\theta<2 \pi .
$$

It has been proved that $\varphi(\theta)$ must satisfy the Theodorsen equation,

$$
\varphi(\theta)-\theta=K\{\log \rho[\varphi(\theta)]\},
$$


which has exactly one continuous solution for $0 \leq \varphi<2 \pi$ when $\rho(\varphi)$ is any continuous positive function of period $2 \pi$. The conjugation operator $K$ is defined by the principal value integral

$$
K f(t)=\frac{1}{2 \pi} \mathrm{PV} \int_{0}^{2 \pi} f(s) \cot \frac{t-s}{2} d s .
$$

There are various non-linear iterative methods to solve (2.1). The dominant computation at each step is a function conjugation, which can be carried out by two FFT's, therefore is $O\left(n \log _{2} n\right)$.

If convergence is achieved, it is typically linear. The global convergence is ensured only if the $\varepsilon$-condition

$$
\varepsilon=\sup _{0 \leq \phi \leq 2 \pi}\left|\rho^{\prime}(\theta) / \rho(\theta)\right|<1
$$

is satisfied. The iteration is likely to diverge even if $\varepsilon$ is slightly greater than 1 . However this difficulty may be alleviated by applying a preliminary transformation which maps the original domain onto a nearly circular region.

Recently Hübner [8] has developed a Newton method to solve the Theodorsen equation and thus has achieved quadratic convergence. This method is based on the rediscovered fact, originally observed by Vertgeim [21], that the Newton correction at each iteration can be viewed as the solution of a Riemann-Hilbert problem.

(ii) Symm's method. If $\Omega_{w}$ is the interior of a unit circle and $\Omega_{z}$ contains the origin, Problem A can be reduced to a Dirichlet problem: it is required to construct a harmonic function $g$ on $\Omega_{z}$ satisfying the boundary conditions

$$
g(z)=-\log |z|, \quad z \in C_{z} .
$$

Once this is done, the function defined by

$$
f(z)=z \exp (g+i h),
$$

where $h$ is the harmonic conjugate of $g$, is the desired solution of Problem A. The method proposed by Symm essentially solves the Dirichlet problem associated with a conformal mapping by an indirect boundary element formulation. More specifically, $g(z)$ is constructed from a single layer potential

$$
g(z)=\int_{C_{z}} \log |z-t| \sigma(t) d t .
$$

The source density $\sigma$ in (2.3) is the solution of the following singular Fredholm integral equation of the first kind, called Symm's equation,

$$
\int_{C_{z}} \log |z-t| \sigma(t) d t=-\log |z|, \quad z \in C_{z} .
$$

In order to solve (2.3) numerically, the boundary has to be discretized and $\sigma$ is interpolated at a set of $n$ points on the boundary. The computation is usually $O\left(n^{3}\right)$. However, Berrut [15] has recently proposed an algorithm which solves Symm's equation in $O\left(n^{2} \log _{2} n\right)$ steps by means of FFT.

(iii) Wegmann's method. It is assumed that $C_{w}$ in Problem A has a parametrization $\eta(s)$ and $\Omega_{z}$ is the unit circle. The problem can be reduced to the determination of a real function $S(\theta)$ satisfying

$$
f\left(e^{i \theta}\right)=\eta[S(\theta)]
$$

where $f\left(e^{i \theta}\right)$ is the boundary value of an analytic function $f(z)$. 
The solution proposed by Wegmann proceeds by iterations. Let $S_{k}(\theta)$ be the approximation of $S(\theta)$ at the $k$-th step; a correction $\sigma(\theta)$ is to be found such that

$$
\eta\left(S_{k}+\sigma\right)=h_{k+1}\left(e^{i \theta}\right)
$$

where $h_{k+1}$ is an analytic function. While it is difficult to determine $\sigma(\theta)$ exactly, the linearized form of $(2.5)$

$$
\eta\left(S_{k}\right)+\eta^{\prime}\left(S_{k}\right) \sigma_{k}=h_{k+1}
$$

is used to obtain an approximation $\sigma_{k}$ of $\sigma$. The updated function $S_{k+1}=S_{k}+\sigma_{k}$ is believed to represent a better approximation of $S(\theta)$.

The method of Wegmann is so far the most efficient for conformal mapping. The efficiency is largely based on the observation that the Newton increment $\sigma_{k}$ in (2.6) is in fact the solution of a Riemann-Hilbert problem. It can be represented explicitly and its numerical computation takes only four FFT's plus several function evaluations. Under suitable assumptions on the smoothness of $C_{w}$, it has been proved that the convergence is locally quadratic.

\section{The CONJUGATION OPERATOR}

Let $\Omega$ be the interior of a unit circle $|z|<1$ and $C$ its boundary. It is assumed that $f(z)=U(x, y)+i V(x, y)$ is an analytic function in $\Omega$ while $U_{0}$ and $V_{0}$ denote $U(0,0)$ and $V(0,0)$, respectively. The Schwarz formula

$$
f(z)=\frac{1}{2 \pi} \int_{0}^{2 \pi} \frac{e^{i t}+z}{e^{i t}-z} U\left(e^{i t}\right) d t+i V_{0}, \quad z \in \Omega,
$$

expresses the function $f(z)$ in terms of the boundary values of its real part.

Now let $z$ tend to a point $z_{0}=e^{i s} \in C$ from inside $\Omega$. The limit of (3.1) as $z \rightarrow e^{i s}$ becomes

$$
\begin{aligned}
U\left(e^{i s}\right)+i V\left(e^{i s}\right) & =\lim _{z \rightarrow e^{i s}} \frac{1}{2 \pi} \int_{0}^{2 \pi} \frac{e^{i t}+z}{e^{i t}-z} \cdot U\left(e^{i t}\right) d t+i V_{0} \\
& =U\left(e^{i s}\right)+\frac{1}{2 \pi} \int_{0}^{2 \pi} \frac{e^{i t}+e^{i s}}{e^{i t}-e^{i s}} \cdot U\left(e^{i t}\right) d t+i V_{0}, \quad 0 \leq s<2 \pi,
\end{aligned}
$$

where the second term on the right hand side is understood to be the Cauchy principal value. The above equation can be further reduced to

$$
V\left(e^{i s}\right)-V_{0}=K \cdot U\left(e^{i s}\right), \quad 0 \leq s<2 \pi,
$$

where the conjugation operator $K$ is defined by [30, 31]

$$
K U\left(e^{i s}\right)=\frac{-i}{2 \pi} \int_{0}^{2 \pi} \frac{e^{i t}+e^{i s}}{e^{i t}-e^{i s}} \cdot U\left(e^{i t}\right) d t, \quad 0 \leq s<2 \pi .
$$

Since the function $g(z)=i f(z)=-V+i U$ is also analytic, its real and imaginary parts satisfy $(3.2)$, hence,

$$
U\left(e^{i s}\right)-U_{0}=-K V\left(e^{i s}\right), \quad 0 \leq s<2 \pi .
$$

It is clear that $K$ is closely related to the theory of analytic functions through (3.2) and (3.4). The relationship is even closer due to the following fact [33]: 
If $U\left(e^{i t}\right), V\left(e^{i t}\right) \in L^{p}(1 \leq p<\infty), 0 \leq t<2 \pi$, satisfy (3.2) or (3.4), then the function $f(z)$ defined by

$$
f\left(e^{i t}\right)=U\left(e^{i t}\right)+i V\left(e^{i t}\right), \quad 0 \leq t<2 \pi
$$

is analytic in $\Omega$.

The above statement actually says that given any real function $U$ (or $V$ ) from function space $L^{p}(1 \leq p<\infty)$ defined on $C$, another function $V$ (or $U$ ) can be constructed by applying the conjugation operator $K$ such that the complex valued function $U+i V$ represents the boundary value of an analytic function in $\Omega$; and equations (3.2) and (3.4) imply each other.

It is worth noting that $K$ takes forms other than (3.3). One of them has already been given by (2.2) which is obtainable from (3.3) by simple algebraic manipulation. The operator defined by $(2.2)$ is sometimes called the Hilbert transform. When $U(t)$ is given by its Fourier series

$$
U\left(e^{i s}\right)=\sum_{k=-\infty}^{+\infty} a_{k} e^{i k s}, \quad 0 \leq s<2 \pi
$$

the conjugation operator is defined by

$$
K U\left(e^{i s}\right)=-i \sum_{k=-\infty}^{+\infty} \operatorname{Sign}(k) a_{k} e^{i k s}, \quad 0 \leq s<2 \pi
$$

where $\operatorname{Sign}(k)=-1,0$ or 1 if $k$ is less than, equal to or greater than zero, respectively. It transpires from (3.5) that $K$ is a bounded linear operator on $L^{2}$ and its norm is $\|K\|_{2}=1$.

\section{Discrete conjugation operator (DCO)}

For numerical implementation, the conjugation operator $K$ has to be approximated. This is achieved by the boundary element method [32]. The formulation proceeds in three steps:

(1) The boundary $C$ is discretized into $n$ line elements with the $n$ nodes being equidistantly distributed along $C$, that is

$$
C=\bigcup_{m=1}^{n} C_{m}
$$

(2) $U$ is represented on each element by a linear interpolation function, namely,

$$
U(Z)=\frac{Z_{m+1}-Z}{Z_{m+1}-Z_{m}} U\left(Z_{m}\right)+\frac{Z-Z_{m}}{Z_{m+1}-Z_{m}} U\left(Z_{m+1}\right)
$$

where $Z \in C_{m}$ and $Z_{m}, Z_{m+1}$ are the end nodes of $C_{m}$;

(3) The function $K U$ is linearly interpolated between the two nodal values on each $C_{m}$ taking (3.3) as the representation of $K$. Since the imaginary value introduced by discretization is meaningless, only the real part of the right 
hand side of (3.3) is retained, hence

$$
\begin{aligned}
& V\left(e^{i s}\right)=K U\left(e^{i s}\right) \\
&=\operatorname{Re}\left[\frac{-1}{2 \pi} \sum_{m=1}^{n} \int_{C_{m}} \frac{e^{i t}+e^{i s}}{e^{i t}-e^{i s}} U\left(e^{i t}\right) \frac{d\left(e^{i t}\right)}{e^{i t}}\right] \\
&=\operatorname{Re}\left\{\frac { - 1 } { 2 \pi } \sum _ { m = 1 } ^ { n } \int _ { Z _ { m } } ^ { Z _ { m + 1 } } \frac { Z + e ^ { i s } } { Z - e ^ { i s } } \left[\frac{Z_{m+1}-Z}{Z_{m+1}-Z_{m}} U\left(Z_{m}\right)\right.\right. \\
&\left.\left.+\frac{Z-Z_{m}}{Z_{m+1}-Z_{m}} U\left(Z_{m+1}\right)\right] \frac{d Z}{Z}\right\} .
\end{aligned}
$$

Let

$$
\begin{aligned}
Z_{m} & =e^{i(2 \pi / n)(m-1)}, \\
v_{k} & =V\left(Z_{k}\right), \quad u_{m}=U\left(Z_{m}\right) ; \quad k, m=1,2, \ldots, n,
\end{aligned}
$$

then

$$
v_{k}=\sum_{m=1}^{n} C_{k m} u_{m}
$$

where

$$
\begin{aligned}
C_{k m}= & \frac{-1}{2 \pi} \operatorname{Re}\left[\int_{Z_{m-1}}^{Z_{m}} \frac{Z+Z_{k}}{Z-Z_{k}} \frac{Z-Z_{m-1}}{Z_{m}-Z_{m-1}} \frac{d Z}{Z}\right] \\
& +\frac{-1}{2 \pi} \operatorname{Re}\left[\int_{Z_{m}}^{Z_{m-1}} \frac{Z+Z_{k}}{Z-Z_{k}} \frac{Z_{m+1}-Z}{Z_{m+1}-Z_{m}} \frac{d Z}{Z}\right] .
\end{aligned}
$$

Denote the space of $n$-tuples of real numbers by $\mathbf{R}^{n}$ and let $\mathbf{U}=\left(u_{1}, u_{2}, \ldots, u_{n}\right)^{T} \in$ $\mathbf{R}^{n}$ and $\mathbf{V}=\left(v_{1}, v_{2}, \ldots, v_{n}\right)^{T} \in \mathbf{R}^{n}$. Then (4.1) can be written in matrix form

$$
\mathbf{V}=\mathbf{C U}
$$

where $\mathbf{C}=\left[C_{k m}\right](k, m=1,2, \ldots, n)$. The matrix $\mathbf{C}$ in (4.3) corresponds to a finite-dimensional approximation of the conjugation operator $K$, and is actually circulant. Therefore, it can be represented by

$$
\mathbf{C}=\operatorname{Circ}\left(c_{1}, c_{2}, \ldots, c_{n}\right) .
$$

The choice of (3.3) rather than the more compact form (2.2) as the representation of $K$ is owing to the fact that the former allows the associated integrals to be carried out explicitly, hence avoids the necessity of numerical integration.

Let $\mathbf{M}=\left(c_{1}, c_{2}, \ldots, c_{n}\right)^{T}$. For any even $n$, careful examination of (4.2) leads to the conclusion that $\mathbf{M}$ is a skew-Hermitian sequence. If the sequence $\mathbf{E}=$ $\left(e_{1}, e_{2}, \ldots, e_{n}\right)^{T}$ contains the eigenvalues of the circulant $\mathbf{C}$, then

$$
\mathbf{E}=\sqrt{n} \mathbf{F}^{*} \mathbf{M}
$$

where $\mathbf{F}^{*}$ is the conjugate transpose of the Fourier matrix defined in the Appendix. Since $\mathbf{M}$ is both real and skew-Hermitian, the theory of discrete Fourier transform [33] predicts that $\mathbf{E}$ is an imaginary and Hermitian sequence, thus,

$$
e_{1}=e_{1+n / 2}=0, \quad e_{i}=-e_{n+2-i}, \quad i=2,3, \ldots, n / 2 .
$$

It is therefore only necessary to study the last $n / 2-1$ eigenvalues of $\mathbf{C}$. Table 1 lists the imaginary parts of the eigenvalues for $n=8,16,32,64$. 
TABLE 1. Eigenvalues of $\mathbf{C}$

\begin{tabular}{cccccc}
\hline$n$ & \multicolumn{5}{c}{$\operatorname{Im}\left(e_{i}\right), i=n / 2+2, \ldots, n$} \\
\hline 8 & -0.6997 & -0.9553 & -0.9978 & & \\
\hline 16 & -0.4037 & -0.7030 & -0.8755 & -0.9568 & -0.9884 \\
& -0.9980 & -0.9999 & & & \\
\hline 32 & -0.2102 & -0.4043 & -0.5706 & -0.7038 & -0.8042 \\
& -0.8761 & -0.9252 & -0.9571 & -0.9770 & -0.9886 \\
& -0.9950 & -0.9981 & -0.9994 & -0.9999 & -1.0000 \\
\hline 64 & -0.1062 & -0.2103 & -0.3102 & -0.4045 & -0.4916 \\
& -0.5708 & -0.6417 & -0.7040 & -0.7581 & -0.8044 \\
& -0.8436 & -0.8762 & -0.9032 & -0.9253 & -0.9430 \\
& -0.9572 & -0.9684 & -0.9770 & -0.9836 & -0.9886 \\
& -0.9923 & -0.9950 & -0.9968 & -0.9981 & -0.9989 \\
& -0.9994 & -0.9997 & -0.9999 & -1.0000 & -1.0000 \\
& -1.0000 & & & & \\
\hline
\end{tabular}

After examining the eigenvalues $e_{i}(i=n / 2+2, \ldots, n)$ for various $n$, the following patterns are recognisable:

(1) $0<\left|e_{i}\right|<1$,

(2) $\left|e_{i-1}\right|<\left|e_{i}\right|$,

(3) $\left|e_{n}\right| \rightarrow 1$ and $\left|e_{2+n / 2}\right| \rightarrow 0$ as $n \rightarrow \infty$,

(4) while there is a large number of $e_{i}$ clustering near unity, only very few $e_{i}$ are distributed close to 0 .

It should be noted that the value $\operatorname{Im}\left(e_{i}\right)=-1$ in Table 1 is only due to rounding down.

\section{Generalised conjugation operator (GCO)}

Let $e_{i}(i=1,2, \ldots, n)$ be the eigenvalues of the Discretized Conjugation Operator $\mathbf{C}$, and $\mathbf{D}=\operatorname{diag}\left(d_{1}, d_{2}, \ldots, d_{n}\right)$, where $d_{i}=2 e_{i} /\left(1+\left|e_{i}\right|^{2}\right)$. The Generalised Conjugation Operator is defined as the circulant matrix

$$
\mathbf{G}=\mathbf{F}^{*} \mathbf{D F} \text {. }
$$

Theorem 5.1. For any $\mathbf{U}=\left(u_{1}, u_{2}, \ldots, u_{n}\right)^{T}$ and $\mathbf{V}_{0}=(b, b, \ldots, b)^{T}$, the finite sequence $\mathbf{V} \in \mathbf{R}^{n}$ given by

$$
\mathbf{V}=\mathbf{G} \mathbf{U}+\mathbf{V}_{0}
$$

is the least square solution of the system

$$
\begin{gathered}
\mathbf{V}=\mathbf{C U}+\mathbf{V}_{0}, \\
\mathbf{C V}=-\mathbf{U}+\mathbf{U}_{0},
\end{gathered}
$$

where $\mathbf{C}$ is the DCO and $\mathbf{U}_{0}=(a, a, \ldots, a)^{T}$ with

$$
a=\frac{1}{n}\left(u_{1}+u_{2}+\cdots+u_{n}\right) .
$$

Similarly, given any $\mathbf{V}=\left(v_{1}, v_{2}, \ldots, v_{n}\right)^{T}$ and $\mathbf{U}_{0}=(a, a, \ldots, a)^{T}$, the finite sequence

$$
\mathbf{U}=-\mathbf{G} \mathbf{V}+\mathbf{U}_{0}
$$


is the least square solution of the system

$$
\begin{gathered}
\mathbf{U}=-\mathbf{C V}+\mathbf{U}_{0}, \\
\mathbf{C U}=\mathbf{V}-\mathbf{V}_{0},
\end{gathered}
$$

where

$$
\mathbf{V}_{0}=(b, b, \ldots, b)^{T} \quad \text { with } \quad b=\frac{1}{n}\left(v_{1}+v_{2}+\cdots+v_{n}\right) .
$$

Proof. The proof is given for only the first case since that for the second is essentially the same. The system of $(5.2 \mathrm{a})$ and $(5.2 \mathrm{~b})$ is written more compactly,

$$
\left[\begin{array}{l}
\mathbf{I} \\
\mathbf{C}
\end{array}\right] \mathbf{V}=\left[\begin{array}{c}
\mathbf{C} \\
-\mathbf{I}
\end{array}\right] \mathbf{U}+\left[\begin{array}{l}
\mathbf{V}_{0} \\
\mathbf{U}_{0}
\end{array}\right]
$$

Obviously, the $2 n \times n$ matrix [ $\mathbf{\mathbf { C }}]$ has full column rank and thus its generalised (M-P) inverse is given by

$$
\left[\begin{array}{l}
\mathbf{I} \\
\mathbf{C}
\end{array}\right]^{\div}=\left\{\left[\begin{array}{ll}
\mathbf{I} & \mathbf{C}^{*}
\end{array}\right]\left[\begin{array}{l}
\mathbf{I} \\
\mathbf{C}
\end{array}\right]\right\}^{-1}\left[\begin{array}{ll}
\mathbf{I} & \mathbf{C}^{*}
\end{array}\right]=\left[\mathbf{I}+\mathbf{C}^{*} \mathbf{C}\right]^{-1}\left[\begin{array}{ll}
\mathbf{I} & \mathbf{C}^{*}
\end{array}\right]
$$

But from the theory of generalised inverse, briefly reviewed in the Appendix, the least square solution of (5.4) is represented by

$$
\begin{aligned}
\mathbf{V} & =\left[\begin{array}{c}
\mathbf{I} \\
\mathbf{C}
\end{array}\right]^{\div}\left(\left[\begin{array}{c}
\mathbf{C} \\
-\mathbf{I}
\end{array}\right] \mathbf{U}+\left[\begin{array}{c}
\mathbf{V}_{0} \\
\mathbf{U}_{0}
\end{array}\right]\right) \\
& =\left(\mathbf{I}+\mathbf{C}^{*} \mathbf{C}\right)^{-1}\left(\mathbf{C}-\mathbf{C}^{*}\right) \mathbf{U}+\left(\mathbf{I}+\mathbf{C}^{*} \mathbf{C}\right)^{-1} \mathbf{V}_{0} \\
& =\mathbf{F}^{*} \mathbf{D F U}+\mathbf{V}_{0}=\mathbf{G} \mathbf{U}+\mathbf{V}_{0} .
\end{aligned}
$$

The proof is completed.

Since, for any real function $U \in L^{p}(1 \leq p<\infty)$ defined in the interior of a unit circle and an arbitrary constant $V_{0}$, there always exists a real function $V$ such that both (3.2) and (3.4) are satisfied, it is natural to expect that the discretized version of this problem will lead to a similar situation, namely that, given finite sequences $\mathbf{U}=\left(u_{1}, u_{2}, \ldots, u_{n}\right)^{T}$ and $\mathbf{V}_{0}=(b, b, \ldots, b)^{T}$, there exists a sequence $\mathbf{V} \in \mathbf{R}^{n}$ satisfying (5.2a) and (5.2b), which are the finite-dimensional counterparts of (3.2) and (3.4). Surprisingly enough, this is not the case, as is shown next.

Theorem 5.2. The system of (5.2a) and (5.2b) is generally not solvable.

Proof. Suppose that, for arbitrary $\mathbf{U}$ and $\mathbf{V}_{0},(5.2 \mathrm{a})$ and $(5.2 \mathrm{~b})$ are satisfied by a V. Then,

$$
\begin{gathered}
\mathbf{C C U}=-\mathbf{U}+\mathbf{U}_{0}, \\
\mathbf{F}^{*} \operatorname{diag}\left(e_{1}^{2}, e_{2}^{2}, \ldots, e_{n}^{2}\right) \mathbf{F U}=\mathbf{F}^{*} \operatorname{diag}(0,-1,-1, \ldots,-1) \mathbf{F U}, \\
\operatorname{diag}\left(e_{1}^{2}, e_{2}^{2}, \ldots, e_{n}^{2}\right)=\operatorname{diag}(0,-1, \ldots,-1)
\end{gathered}
$$

which leads to $e_{1}=0$ and $e_{j}= \pm i$ for $j=2,3, \ldots, n$. But for any finite $n$, the eigenvalues $e_{j}(j=1,2, \ldots, n)$ of $\mathbf{C}$ do not satisfy the above condition, therefore the system of $(5.2 \mathrm{a})$ and $(5.2 \mathrm{~b})$ is not generally solvable.

The above theorem actually explains the rationale behind the introduction of GCO and the following one is concerned with the norm of $\mathbf{G}$. 
Theorem 5.3. The $l_{2}$ norm of the generalised conjugation operator $\|\mathbf{G}\|_{2}$ is less than 1 unless the discrete conjugation operator has an eigenvalue of modulus equal to 1 , in which case $\|\mathbf{G}\|_{2}=1$.

Proof. For a real $n \times n$ matrix $\mathbf{A}$, it is known that

$$
\|\mathbf{A}\|_{2}=\left[\rho\left(\mathbf{A}^{T} \mathbf{A}\right)\right]^{1 / 2}
$$

where $\rho\left(\mathbf{A}^{T} \mathbf{A}\right)$ is the spectral radius of $\mathbf{A}^{T} \mathbf{A}$. By the definition of $\mathbf{G}$,

$$
\|\mathbf{G}\|_{2}=\max \left(\frac{2\left|e_{i}\right|}{1+\left|e_{i}\right|^{2}}\right)
$$

where $e_{i}(i=1,2, \ldots, n)$ are the eigenvalues of the discrete conjugation operator C. Obviously,

$$
\frac{2\left|e_{i}\right|}{1+\left|e_{i}\right|^{2}} \leq 1
$$

The equality holds only when $\left|e_{i}\right|=1$. The proof is completed.

\section{A NEW ALGORithm For NCM BASED on GCO}

It is required to construct an analytic function $f(z)=U+i V$ which conformally maps the interior of the unit disc in the $z$-plane onto a simply-connected domain $\Omega$ with boundary $\mathbf{C}_{w}$ in the $w$-plane. The first step toward the solution is to determine the boundary value $f\left(e^{i s}\right)(0 \leq s \leq 2 \pi)$ of the mapping function $f(z)$. This is equivalent to finding two real value functions $U\left(e^{i s}\right)$ and $V\left(e^{i s}\right)$, satisfying (3.2) and (3.4), such that $U\left(e^{i s}\right)+i V\left(e^{i s}\right) \in C_{w}$ for $0 \leq s \leq 2 \pi$. Once its boundary value is known, the desired analytic function $f(z)$ is given by the Cauchy integral formula

$$
f(z)=\frac{1}{2 \pi i} \int_{C_{z}} \frac{f\left(e^{i t}\right)}{e^{i t}-z} d\left(e^{i t}\right)
$$

where $C_{z}$ is the unit circle.

According to the discretization scheme described in the last section, $U\left(e^{i s}\right)$ and $V\left(e^{i s}\right)$ can be approximately represented by two finite sequences $\mathbf{U}=$ $\left(u_{1}, u_{2}, \ldots, u_{n}\right)^{T}$ and $\mathbf{V}=\left(v_{1}, v_{2}, \ldots, v_{n}\right)^{T}$, where $u_{j}=U\left(w^{j-1}\right)$ with $w=e^{i 2 \pi / n}$. For an arbitrary $\mathbf{X}=\left(x_{1}, x_{2}, \ldots, x_{n}\right)^{T}$, an $\mathbf{X}_{0}=(a, a, \ldots, a)^{T}$ is defined with $a=$ $\left(x_{1}+x_{2}+\cdots+x_{n}\right) / n$. The problem of conformal mapping can be numerically solved by finding two sequences $\mathbf{U}$ and $\mathbf{V}$ such that $u_{j}+i v_{j} \in C_{w}$ for $j=1,2, \ldots, n$ and satisfying the discretized forms of (3.2) and (3.4), namely

$$
\begin{gathered}
\mathbf{V}-\mathbf{V}_{0}=\mathbf{C U}, \\
\mathbf{U}-\mathbf{U}_{0}=-\mathbf{C V} .
\end{gathered}
$$

For any $0<r<1$, if $z=r e^{i s}(0<s<2 \pi)$, (6.1) becomes

$$
f\left(r e^{i s}\right)=\frac{1}{2 \pi i} \int_{C_{z}} \frac{f\left(e^{i t}\right)}{e^{i t}-r e^{i s}} d\left(e^{i t}\right) .
$$

Let $\boldsymbol{\Phi}=\left(\phi_{1}, \phi_{2}, \ldots, \phi_{n}\right)^{T}$ and $\boldsymbol{\Psi}(r)=\left(\psi_{1}(r), \psi_{2}(r), \ldots, \psi_{n}(r)\right)^{T}$, where $\phi_{j}=$ $f\left(w^{j-1}\right)$ and $\psi_{j}(r)=f\left(r w^{j-1}\right)$. The discretization of (6.3) leads to

$$
\boldsymbol{\Psi}(r)=\mathbf{A}(r) \boldsymbol{\Phi}
$$

where $\mathbf{A}(r)$ is again a circulant matrix which varies with $r$. 
Given a vector space $\mathbf{R}^{n}$, a vector in the direct product space $\mathbf{R}^{n} \times \mathbf{R}^{n}$ is denoted by $(\mathbf{X}, \mathbf{Y})$ where $\mathbf{X} \in \mathbf{R}^{n}$ and $\mathbf{Y} \in \mathbf{R}^{n}$. Let $\Gamma$ be a plane curve. The $\Gamma$-projection operator $P_{\Gamma}: \mathbf{R}^{n} \times \mathbf{R}^{n} \rightarrow \mathbf{R}^{n} \times \mathbf{R}^{n}$ is defined by

$$
P_{\Gamma}:(\mathbf{X}, \mathbf{Y}) \rightarrow(\mathbf{U}, \mathbf{V})
$$

where $\left(u_{i}, v_{i}\right)$ is the nearest point on $\Gamma$ corresponding to the point $\left(x_{i}, y_{i}\right)$ for $i=$ $1,2, \ldots, n$.

A new algorithm for NCM is proposed.

INPUT The number of nodes $n$; the maximum number of iterations $N$; the number of interior circles $m$, on which the mapping function is to be evaluated; the radii of the circles $r_{1}, r_{2}, \ldots, r_{m}$; the initial approximations $\mathbf{U}$ and $\mathbf{V}$; the tolerance $T$; the representation of the boundary $\Gamma$.

OUTPUT $\boldsymbol{\Phi}=\mathbf{U}+i \mathbf{V}, \boldsymbol{\Psi}\left(r_{1}\right), \boldsymbol{\Psi}\left(r_{2}\right), \ldots, \boldsymbol{\Psi}\left(r_{m}\right)$.

Step 1: Calculate the generalised conjugation operator $\mathbf{G}$.

Step 2: For $i=1$ to $N$, do steps 3-7.

Step 3: Set $\overline{\mathbf{U}}=\mathbf{U}$ and $\overline{\mathbf{V}}=\mathbf{V}$.

Step 4: Set $\mathbf{U}=\mathbf{D}_{1}\left(-\mathbf{G} \overline{\mathbf{V}}+\overline{\mathbf{U}}_{0}\right)+\left(\mathbf{I}-\mathbf{D}_{1}\right) \overline{\mathbf{U}}$, $\mathbf{V}=\mathbf{D}_{2}\left(\mathbf{G} \overline{\mathbf{U}}+\overline{\mathbf{V}}_{0}\right)+\left(\mathbf{I}-\mathbf{D}_{2}\right) \overline{\mathbf{V}}$

where $\mathbf{D}_{1}$ and $\mathbf{D}_{2}$ are diagonal matrices whose elements have values in the interval $[0,1])$.

Step 5: Set $(\mathbf{U}, \mathbf{V})=P_{\Gamma}(\mathbf{U}, \mathbf{V})$,

Set $e=\frac{1}{2}(\|\mathbf{U}-\overline{\mathbf{U}}\|+\|\mathbf{V}-\overline{\mathbf{V}}\|)$.

Step 6: If $e<T$ then go to Step 8.

Step 7: Continue.

Step 8: For $i=1$ to $m$ do Steps $9-10$.

Step 9: Calculate $\mathbf{A}\left(r_{i}\right)$.

Step 10: Set $\boldsymbol{\Psi}\left(r_{i}\right)=\mathbf{A}\left(r_{i}\right) \boldsymbol{\Phi}$.

Step 11: Output $\boldsymbol{\Phi}, \boldsymbol{\Psi}\left(r_{1}\right), \boldsymbol{\Psi}\left(r_{2}\right), \ldots, \boldsymbol{\Psi}\left(r_{m}\right)$.

Stop.

The dominant computation in the algorithm is performed in the loop consisting of Steps 3-7 which calculate the boundary value of the mapping function iteratively. Once this is done, Steps 9-10 evaluate the mapping function on the desired internal circles. The computational cost is $O\left(n \log _{2} n\right)$ by means of FFT.

The iteration used in Steps $3-7$ is actually a relaxation method. This can be seen more clearly when the formulas in step 4 are rewritten,

$$
\begin{gathered}
\mathbf{U}^{(i)}=\mathbf{U}^{(i-1)}+\mathbf{D}_{1}\left(-\mathbf{G} \mathbf{V}^{(i-1)}+\mathbf{U}_{0}^{(i-1)}-\mathbf{U}^{(i-1)}\right), \\
\mathbf{V}^{(i)}=\mathbf{V}^{(i-1)}+\mathbf{D}_{2}\left(\mathbf{G} \mathbf{U}^{(i-1)}+\mathbf{V}_{0}^{(i-1)}-\mathbf{V}^{(i-1)}\right) .
\end{gathered}
$$

The matrices $\mathbf{D}_{1}$ and $\mathbf{D}_{2}$ consist of relaxation parameters and the terms following them can be viewed as residuals.

While theoretical optimum values of $\mathbf{D}_{1}$ and $\mathbf{D}_{2}$ are difficult to identify, several relaxation matrices have been tested in implementing the algorithm. The following values for $\mathbf{D}_{1}$ and $\mathbf{D}_{2}$ were found effective,

$$
\begin{aligned}
& \mathbf{D}_{1}=\operatorname{diag}\left(\cos ^{2} \theta_{1}, \cos ^{2} \theta_{2}, \ldots, \cos ^{2} \theta_{n}\right), \\
& \mathbf{D}_{2}=\operatorname{diag}\left(\sin ^{2} \theta_{1}, \sin ^{2} \theta_{2}, \ldots, \sin ^{2} \theta_{n}\right),
\end{aligned}
$$

where $\theta_{j}$ is the angle between the $U$-axis and the tangent of $\Gamma$ at the point $\left(u_{j}^{(i-1)}, v_{j}^{(i-1)}\right)$. When $\Gamma$ is not differentiable at $\left(u_{j}^{(i-1)}, v_{j}^{(i-1)}\right)$, the mean value of the left and the right limits of $\cos ^{2} \theta_{j}$ is used. 
Although the algorithm is designed for simply-connected domains, a similar strategy should be applicable to doubly-connected domains as well, particularly to those with a boundary at infinity.

\section{Convergence Study}

According to Riemann's mapping theorem, infinitely many mapping functions $f(z)$ exist. The mappings become unique when $f(0)$ and $f(1)$ are specified. But this is deliberately not done so that the algorithm may quickly converge to a solution which is closest to the initial approximation. However, in order to study the convergence, $f(0)$ is specified for simplicity. Now let $f(0)=a+i b, \mathbf{U}_{0}=(a, a, \ldots, a)^{T}$, $\mathbf{V}_{0}=(b, b, \ldots, b)^{T}$, and $(\mathbf{U}, \mathbf{V})$ be a numerical solution. Then according to Theorem 5.1,

$$
\begin{gathered}
\mathbf{U}=-\mathbf{G} \mathbf{V}+\mathbf{U}_{0}, \\
\mathbf{V}=\mathbf{G} \mathbf{U}+\mathbf{V}_{0}
\end{gathered}
$$

within truncation accuracy. For any initial approximation $\left(\mathbf{U}_{0}, \mathbf{V}_{0}\right)$, with $\mathbf{D}_{1}$ and $\mathbf{D}_{2}$ taken, for simplicity, as unit matrices, the algorithm proceeds by evaluating

$$
\begin{gathered}
\mathbf{X}^{i}=-\mathbf{G} \mathbf{V}^{i-1}+\mathbf{U}_{0}, \\
\mathbf{Y}^{i}=\mathbf{G} \mathbf{U}^{i-1}+\mathbf{V}_{0}
\end{gathered}
$$

and

$$
\left(\mathbf{U}^{i}, \mathbf{V}^{i}\right)=P_{\Gamma}\left(\mathbf{X}^{i}, \mathbf{Y}^{i}\right), \quad i=1,2, \ldots
$$

For $(\mathbf{X}, \mathbf{Y}) \in \mathbf{R}^{n} \times \mathbf{R}^{n}\left(\mathbf{X}, \mathbf{Y} \in \mathbf{R}^{n}\right)$, the $l_{2}$ norm of $(\mathbf{X}, \mathbf{Y})$ is given by

$$
\|(\mathbf{X}, \mathbf{Y})\|_{2}=\left(\|\mathbf{X}\|_{2}^{2}+\|\mathbf{Y}\|_{2}^{2}\right)^{1 / 2} .
$$

Conjecture. Within truncation error, the proposed algorithm converges in a global sense.

Proof of plausibility. Subtracting (7.1a) and (7.1b) from (7.2a) and (7.2b), respectively gives

$$
\begin{gathered}
\mathbf{X}^{i}-\mathbf{U}=-\mathbf{G}\left(\mathbf{V}^{i-1}-\mathbf{V}\right), \\
\mathbf{Y}^{i}-\mathbf{V}=\mathbf{G}\left(\mathbf{U}^{i-1}-\mathbf{U}\right) .
\end{gathered}
$$

As noted in Section 4, all eigenvalues of a discrete conjugation operator appear to be less than 1 in magnitude, which implies that $\|\mathbf{G}\|_{2}<1$ by Theorem 5.3. Thus,

$$
\left\|\mathbf{X}^{i}-\mathbf{U}\right\|_{2}<\left\|\mathbf{V}^{i-1}-\mathbf{V}\right\|_{2}, \quad\left\|\mathbf{Y}^{i}-\mathbf{V}\right\|_{2}<\left\|\mathbf{U}^{i-1}-\mathbf{U}\right\|_{2} .
$$

From (7.4) it follows that

$$
\left\|\left(\mathbf{X}^{i}, \mathbf{Y}^{i}\right)-(\mathbf{U}, \mathbf{V})\right\|_{2}<\left\|\left(\mathbf{U}^{i-1}, \mathbf{V}^{i-1}\right)-(\mathbf{U}, \mathbf{V})\right\|_{2} .
$$

On the other hand, by referring to the definition of the operator $P_{\Gamma}$, the following assertion should be plausible,

$$
\left\|\left(\mathbf{U}^{i}, \mathbf{V}^{i}\right)-(\mathbf{U}, \mathbf{V})\right\|_{2}<\left\|\left(\mathbf{X}^{i}, \mathbf{Y}^{i}\right)-(\mathbf{U}, \mathbf{V})\right\|_{2} .
$$

By (7.5) and (7.6),

$$
\left\|\left(\mathbf{U}^{i}, \mathbf{V}^{i}\right)-(\mathbf{U}, \mathbf{V})\right\|_{2}<\left\|\left(\mathbf{U}^{i-1}, \mathbf{V}^{i-1}\right)-(\mathbf{U}, \mathbf{V})\right\|_{2} .
$$


Therefore, starting from any approximation $\left(U^{0}, V^{0}\right)$, the algorithm produces a sequence $\left(\mathbf{U}^{i}, \mathbf{V}^{i}\right)(i=1,2, \ldots)$ with the property that $\left(\mathbf{U}^{i}, \mathbf{V}^{i}\right)$ is always a better approximation than $\left(\mathbf{U}^{i-1}, \mathbf{V}^{i-1}\right)$. The conjecture is seen to be highly tenable.

There are three difficulties which prevent this conjecture from being a theorem. First, the empirical relation (7.5) is not proved. Second, (7.6) is purely based on intuitive rather than formal logic. Third, there is no guarantee that (7.7) implies

$$
\left\|\left(\mathbf{U}^{i}, \mathbf{V}^{i}\right)-(\mathbf{U}, \mathbf{V})\right\|_{2} \rightarrow 0 \quad \text { as } i \rightarrow \infty .
$$

A series of demanding problems were designed to test the algorithm and no divergence has occurred.

\section{IMPLEMENTATION AND NUMERICAL TESTS}

The algorithm presented above has been implemented in FORTRAN. The code consists of the main program and 16 subroutines together with three NAG library functions for FFT analysis. Most of the complexity is related to the crowding phenomenon, also called Geneva effect, a notorious difficulty for NCM. Fornberg [25] reported a case in which, for a set of uniformly distributed points, the density of the mapped images varies by as much as a factor of 400 along the boundary. For one of the problems solved by the present method, the maximum density is $0.6570554 \times 10^{6}$ times the minimum.

A wide range of problems were designed to test the robustness of the program. Mapping was carried out in the six cases illustrated in Figs. 1-6 and some numerical results for these examples are listed in Table 2. The meaning of the symbols appearing in Table 2 is:

$$
n=\text { number of nodes. }
$$

$m=$ number of iterations.

$\Delta_{1}=l_{1}$ norm of the improvement by the first iteration.

$\Delta_{m}=l_{1}$ norm of the improvement by the last iteration.

$$
R=l_{1} \text { norm of the residue vector }\left(\mathbf{U}^{m}-\mathbf{C V}^{m}-\mathbf{U}_{0}^{m}, \mathbf{V}^{m}+\mathbf{C} \mathbf{U}^{m}-\mathbf{V}_{0}^{m}\right) \text {. }
$$

where the $l_{1}$ norm of a vector $(\mathbf{X}, \mathbf{Y}) \in \mathbf{R}^{n} \times \mathbf{R}^{n}$ is defined by

$$
\|(\mathbf{X}, \mathbf{Y})\|=\frac{1}{2 n}\left(\left|\mathbf{X}_{1}\right|+\left|\mathbf{X}_{2}\right|+\cdots+\left|\mathbf{X}_{n}\right|+\left|\mathbf{Y}_{1}\right|+\left|\mathbf{Y}_{2}\right|+\cdots+\left|\mathbf{Y}_{n}\right|\right) .
$$

It is worth noting that all the examples except example 2 have non-smooth boundaries. A particular promising feature of the program is that it can cope with domains with cracks as shown in Figs. 5 and 6.

TABle 2. Performance of the numerical algorithm

\begin{tabular}{lcclcc}
\hline No. & $n$ & $m$ & $\Delta_{1}$ & $\Delta_{m}$ & $R$ \\
\hline 1 & 512 & 41 & $0.10104669 \times 10^{0}$ & $0.92845586 \times 10^{-5}$ & $0.26434862 \times 10^{-3}$ \\
2 & 512 & 10 & $0.70355799 \times 10^{-1}$ & $0.66744256 \times 10^{-5}$ & $0.27908080 \times 10^{-4}$ \\
3 & 512 & 50 & $0.71911930 \times 10^{-1}$ & $0.50901045 \times 10^{-4}$ & $0.63661337 \times 10^{-3}$ \\
4 & 512 & 32 & $0.84973517 \times 10^{-1}$ & $0.94241229 \times 10^{-5}$ & $0.89792556 \times 10^{-2}$ \\
5 & 512 & 20 & $0.12951817 \times 10^{-1}$ & $0.94362245 \times 10^{-5}$ & $0.63127624 \times 10^{-2}$ \\
6 & 512 & 37 & $0.89485350 \times 10^{-1}$ & $0.88609177 \times 10^{-5}$ & $0.19803488 \times 10^{-2}$ \\
\hline
\end{tabular}


TABLE 3. Residual error

\begin{tabular}{ccccc}
\hline & \multicolumn{2}{c}{ Example 1} & & Example 2 \\
\cline { 2 - 5 }$n$ & $m$ & $R$ & $m$ & $R$ \\
\hline 64 & 36 & $0.60685029 \mathrm{E}-02$ & 11 & $0.17620477 \mathrm{E}-02$ \\
& 29 & $0.60702471 \mathrm{E}-02$ & 9 & $0.17619187 \mathrm{E}-02$ \\
& 18 & $0.61014670 \mathrm{E}-02$ & 7 & $0.17669442 \mathrm{E}-02$ \\
& 8 & $0.71021459 \mathrm{E}-02$ & 5 & $0.18240110 \mathrm{E}-02$ \\
\hline 128 & 50 & $0.23762966 \mathrm{E}-02$ & 18 & $0.47801809 \mathrm{E}-03$ \\
& 39 & $0.23739123 \mathrm{E}-02$ & 10 & $0.47112142 \mathrm{E}-03$ \\
& 25 & $0.24007058 \mathrm{E}-02$ & 7 & $0.46280896 \mathrm{E}-03$ \\
& 8 & $0.45294778 \mathrm{E}-02$ & 5 & $0.56127843 \mathrm{E}-03$ \\
\hline 256 & 50 & $0.70615093 \mathrm{E}-3$ & 21 & $0.11968090 \mathrm{E}-03$ \\
& 35 & $0.70853861 \mathrm{E}-3$ & 10 & $0.11505557 \mathrm{E}-03$ \\
& 19 & $0.85164402 \mathrm{E}-3$ & 7 & $0.13063008 \mathrm{E}-03$ \\
& 6 & $0.22042739 \mathrm{E}-2$ & 5 & $0.50775510 \mathrm{E}-03$ \\
\hline 512 & 50 & $0.25787434 \mathrm{E}-03$ & 13 & $0.29034335 \mathrm{E}-04$ \\
& 41 & $0.26434862 \mathrm{E}-03$ & 10 & $0.27908080 \mathrm{E}-04$ \\
& 21 & $0.51263787 \mathrm{E}-03$ & 7 & $0.10712332 \mathrm{E}-03$ \\
& 6 & $0.19963725 \mathrm{E}-02$ & 5 & $0.56999157 \mathrm{E}-03$ \\
\hline
\end{tabular}

Although theoretically any conformal mapping problem should have infinitely many exact solutions, its discretized version is generally not solvable as suggested by Theorem 5.2. It has to be noted that the difficulty is at the heart of the problem, therefore it cannot be overcome by using any finite degree of discretization. This is particularly true for domains with distorted shapes or non-smooth boundaries due to the crowding effect which has been the subject of much recent study [34, 35]. Thus, the minimum residue vector of an NCM problem is typically not zero. A vector which minimises the residue vector is accepted as a solution of the problem.

The residuals of test examples 1 and 2 are listed in Table 3, for various $n$ and $m$. The notation is the same as in Table 2. Obviously, the residual errors depend on both the numbers of nodes and iterations and the complexity of the boundary geometry. It is generally not possible to estimate the overall amount of computational effort required to achieve a given accuracy for domains with non-smooth boundaries because the number of iterations is closely related to the boundary complexity which itself cannot be meaningfully quantified by any method known to the authors. This is particularly true for domains with cracks.

For any problem, there is a minimum residual error $R_{m}$ corresponding to each boundary discretization. The program will reach $R_{m}$ after a certain number of iterations. Further iterations would not improve the result. It is discernible from Table 3 that the minimum residuals are roughly $O\left(1 / n^{3 / 2}\right) \sim O\left(1 / n^{2}\right)$. The convergence speed and the solution accuracy are significantly higher if the boundary is smoother. It is quite remarkable that for example 2 the programme takes only 7-10 iterations to reach the minimum residual error. 


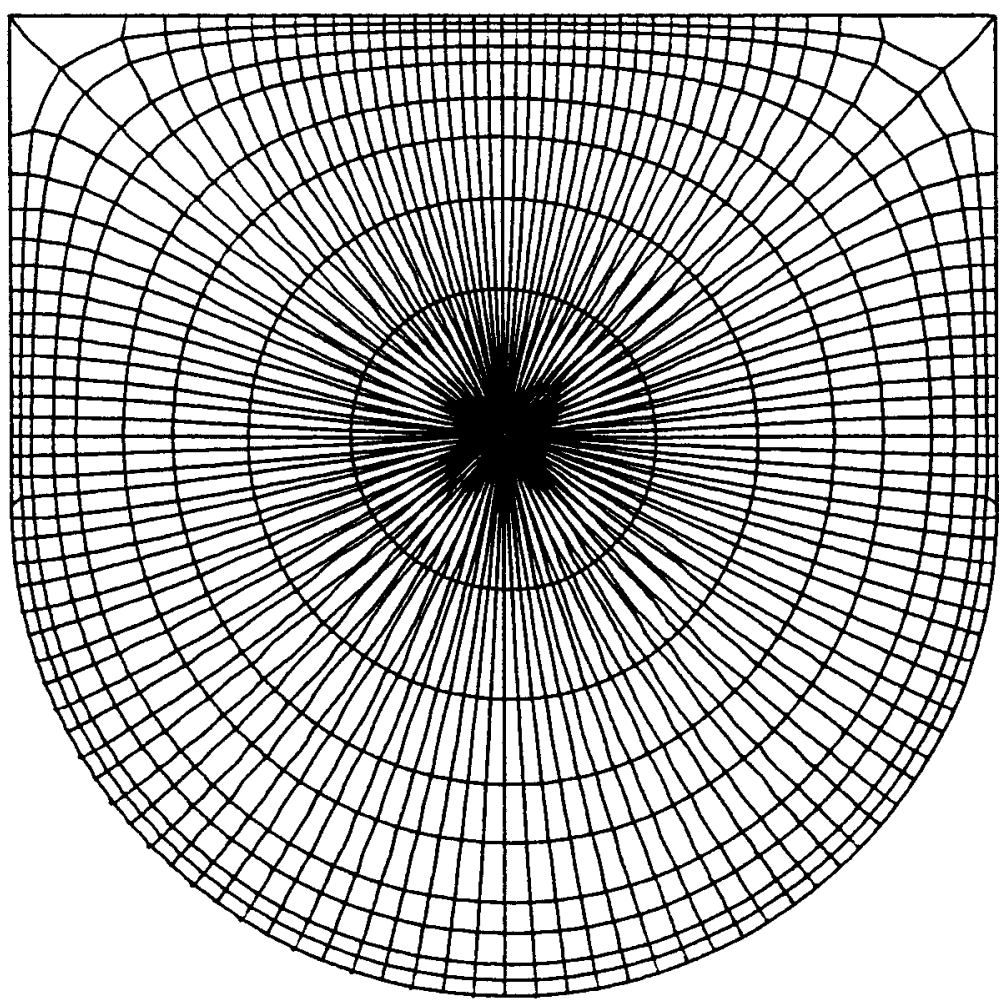

FiguRE 1. Mapping example 1: domain with non-smooth boundary

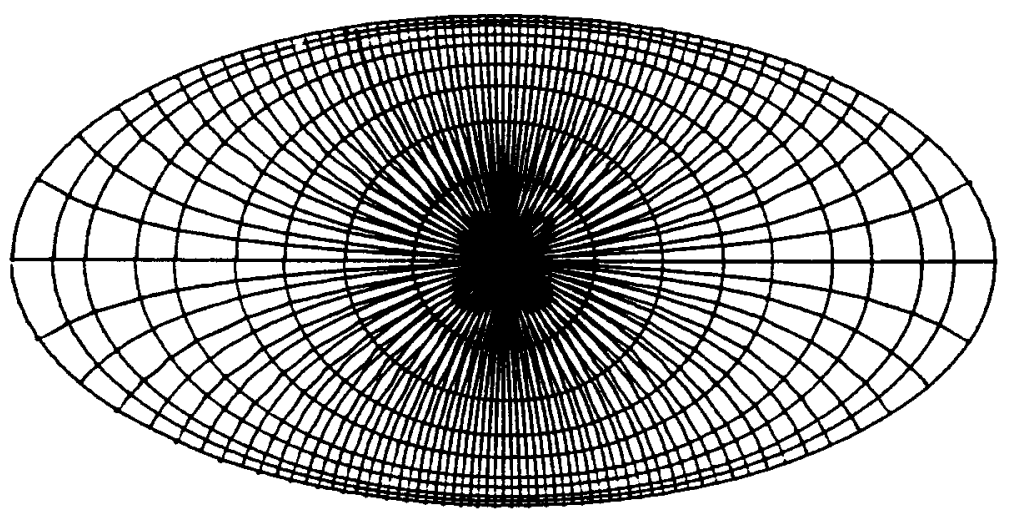

FIGURE 2. Mapping example 2: elliptical domain 


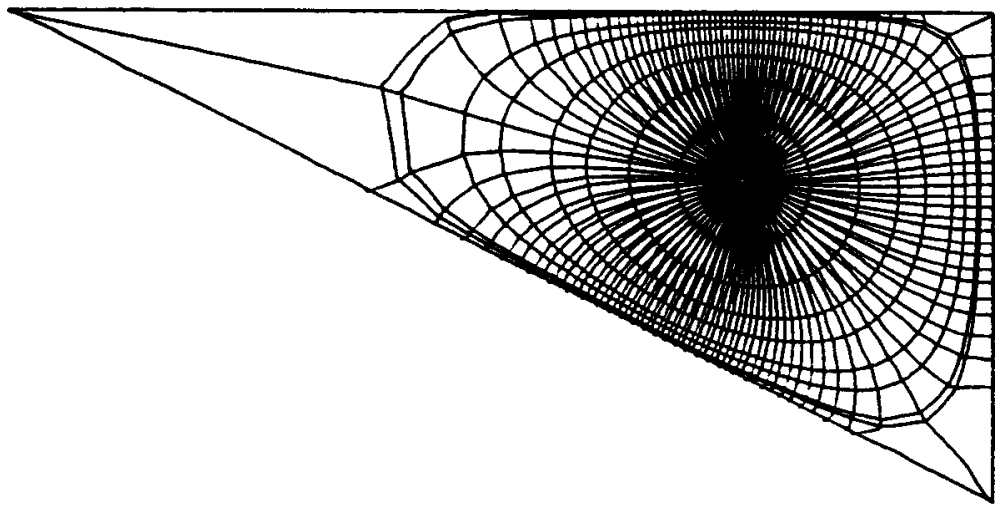

Figure 3. Mapping example 3: triangular domain

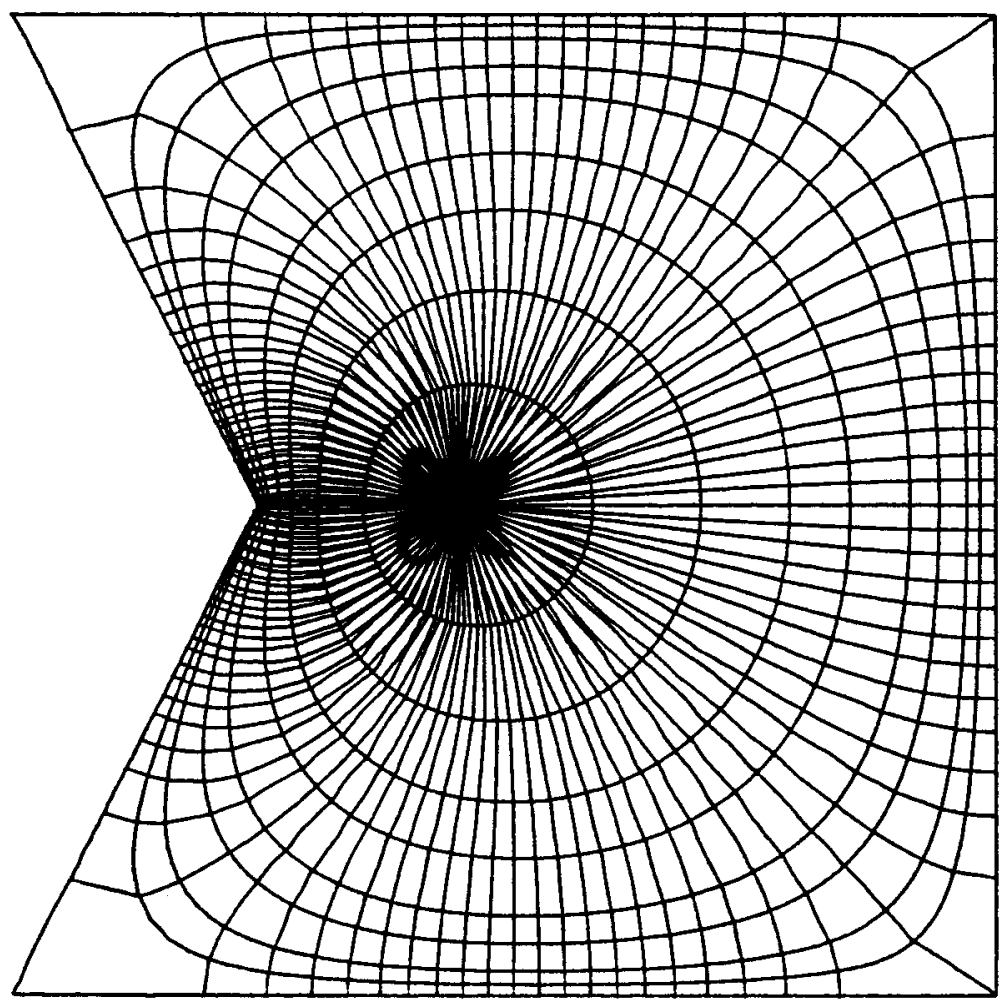

FiguRE 4. Mapping example 4: boundary with re-entrant corner 


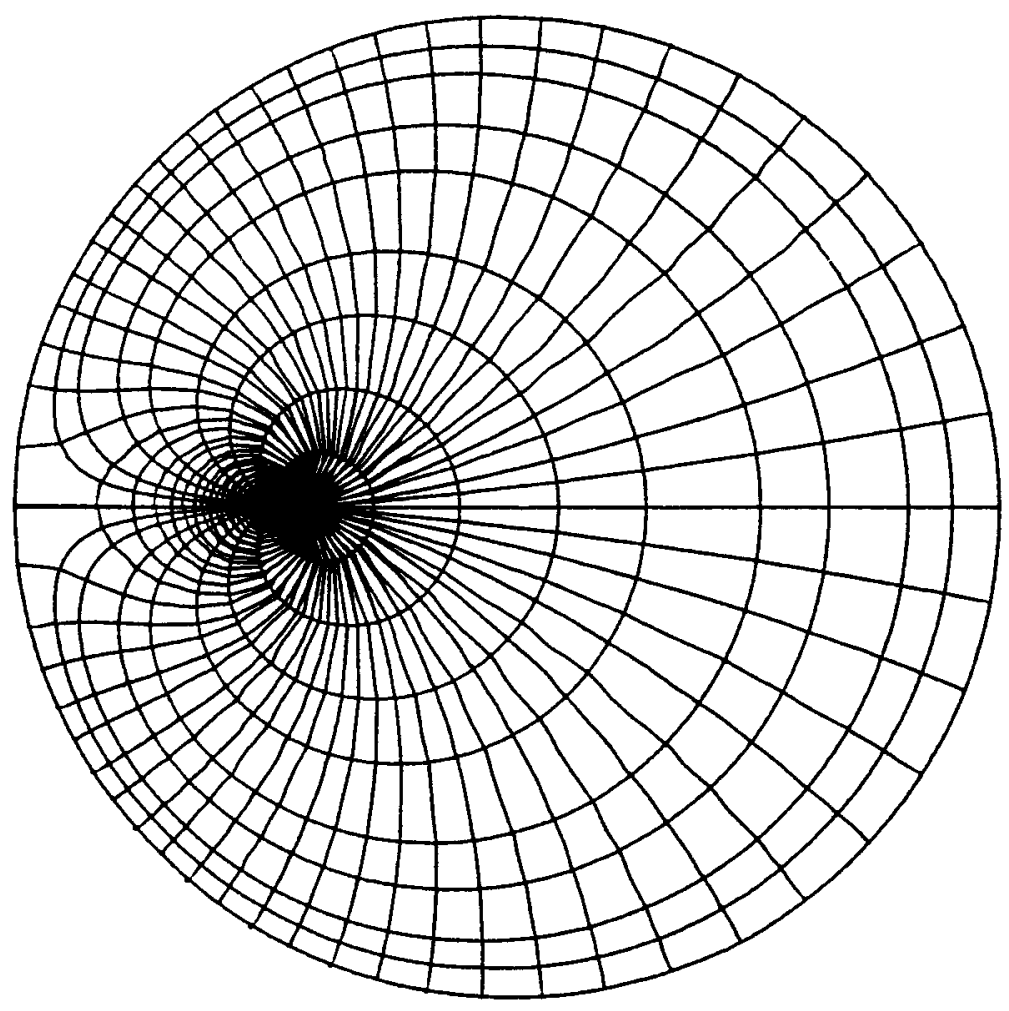

Figure 5. Mapping example 5: circular domain with a crack 


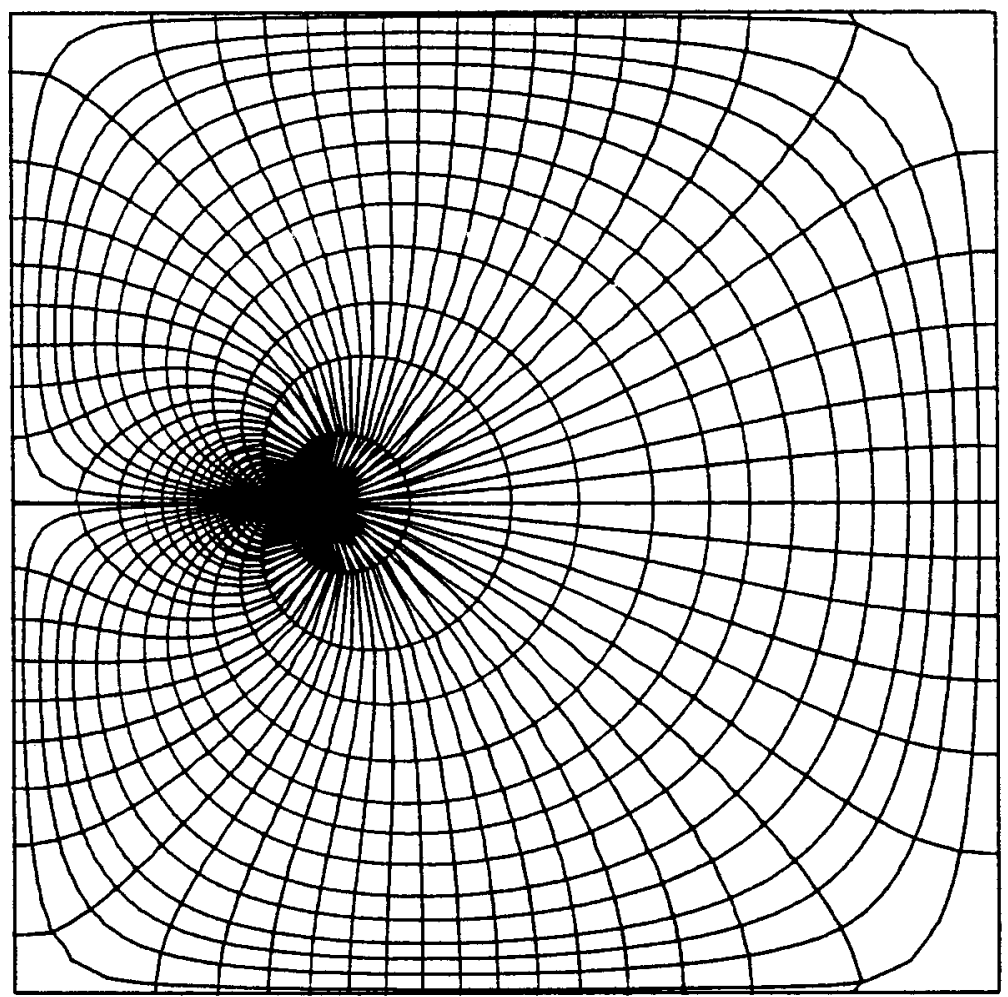

FiguRE 6. Mapping example 6: square domain with a crack

\section{Comparison With previous algorithms}

Most of the algorithms in the literature work only for domains bounded by a restricted class of curves, that is, star-like, smooth, Hölder-continuously differentiable or Jordan curves, and none of them have achieved global convergence. However the present algorithm can be freely applied to any simply-connected domain, and appears to converge globally. Owing to the inherent complexity of NCM problems, this conjecture may never be mathematically proved, but it is well supported by the trial examples.

Regarding computational efficiency, the present algorithm is also superior to earlier ones. It is generally accepted that the most efficient algorithm up to now is based on Wegmann's method and this is believed to converge 7.5 times faster than the one based on Fornberg's method [31]. Each step in Wegmann's algorithm requires two complex FFT's and two real FFT's in addition to evaluating five elementary functions of sine or cosine type. If $n$ is not too large, $n<5000$ for instance, the effect of evaluating a trigonometric function at $n$ points is usually not less than the effort to perform an FFT on an $n$-dimensional real vector. Thus for Wegmann's method, each iteration is computationally equivalent to about 11 real FFT's. In contrast, a step in the present algorithm takes only four real FFT's. The speeds of convergence for the two algorithms seem to be comparable based on our 
limited numerical tests. It is therefore suggested that the algorithm in this work should be more efficient than Wegmann's method.

\section{APPENDIX}

The subjects of circulant matrix and generalised inverse are briefly reviewed for reference. A more detailed treatment is given by Davis [36].

Circulant matrix. A matrix of the form

$$
\mathbf{C}=\operatorname{Circ}\left(c_{1}, c_{2}, \ldots, c_{n}\right)=\left[\begin{array}{cccc}
c_{1} & c_{2} & \cdots & c_{n} \\
c_{n} & c_{1} & \cdots & c_{n-1} \\
\vdots & \vdots & \vdots & \vdots \\
c_{2} & c_{3} & \cdots & c_{1}
\end{array}\right]
$$

is called a circulant matrix, or simply a circulant for short. It is known that $\mathbf{C}$ is diagonalized by a Fourier matrix, namely,

$$
\mathbf{C}=\mathbf{F}^{*} \mathbf{D F}
$$

where the Fourier matrix $\mathbf{F}$ and the diagonal matrix $\mathbf{D}$ are defined by

$$
\begin{gathered}
F_{i j}=\frac{1}{\sqrt{n}} \exp \left[\frac{2 \pi}{n} \sqrt{-1}(i-1)(j-1)\right], \\
\mathbf{D}=\operatorname{diag}\left(d_{1}, d_{2}, \ldots, d_{n}\right)=\left[\begin{array}{cccc}
d_{1} & 0 & \cdots & 0 \\
0 & d_{2} & \cdots & 0 \\
\vdots & \vdots & \vdots & \vdots \\
0 & 0 & \cdots & d_{n}
\end{array}\right]
\end{gathered}
$$

and $\mathbf{F}^{*}$ denotes the conjugate transpose of $\mathbf{F}$. Obviously the diagonal elements of $\mathbf{D}$ represent the eigenvalues of $\mathbf{C}$, which are related to the elements of $\mathbf{C}$ by the following equation,

$$
\mathbf{L}=\sqrt{n} \mathbf{F}^{*} \mathbf{M}
$$

in which

$$
\mathbf{L}=\left(d_{1}, d_{2}, \ldots, d_{n}\right)^{T}, \quad \mathbf{M}=\left(c_{1}, c_{2}, \ldots, c_{n}\right)^{T} .
$$

Since the Fourier matrix is unitary, that is, $\mathbf{F}^{-1}=\mathbf{F}^{*}$, the effort to invert a circulant matrix is practically trivial. More explicitly, if $\mathbf{C}=\mathbf{F}^{*} \mathbf{D F}$, then $\mathbf{C}^{-1}=\mathbf{F}^{*} \mathbf{D}^{-1} \mathbf{F}$. The manipulation of circulant matrices is completely reduced to that of the Fourier matrix. Thus, for instance, the solution of a linear system of algebraic equations requires only $n \log _{2} n$ operations by means of FFT if the associated matrix is a circulant. In contrast, the computation involved in solving a general linear system is typically $O\left(n^{3}\right)$.

Generalised inverse. A linear system $\mathbf{A X}=\mathbf{Y}$ is generally not solvable when $\mathbf{A}$ is either a singular square or a rectangular matrix. If the case arises, an approximate solution such as the least square solution is usually desirable. It is mainly the problems of this sort which have prompted the investigation on the so called generalised inverses of matrices.

Let $\mathbf{A}$ be an $m \times n$ matrix and $\mathbf{B}$ an $n \times m$ matrix, then $\mathbf{B}$ is called a generalised inverse of $\mathbf{A}$ if it satisfies any of the following conditions:

(1) $\mathbf{A B A}=\mathbf{A}$,

(2) $\mathbf{B A B}=\mathbf{B}$, 
(3) $(\mathbf{A B})^{*}=\mathbf{A B}$,

(4) $(\mathbf{B A})^{*}=\mathbf{B A}$.

A matrix with all the four properties is called a Moore-Penrose or M-P inverse of $\mathbf{A}$, and it is denoted by $\mathbf{A} \div$. It has been proved that there always exists a unique M-P inverse for an arbitrary matrix. There are actually recursive algorithms for the numerical computations of M-P inverses.

What makes M-P inverses useful is the fact that the vector $\mathbf{X}=\mathbf{A} \div \mathbf{Y}$ is the least square solution with minimum $l_{2}$ norm of the linear system:

$$
\mathbf{A X}=\mathbf{Y} \text {. }
$$

The general solution of (A.2) is given by

$$
\mathbf{X}=\mathbf{A} \div \mathbf{Y}+(\mathbf{I}-\mathbf{A} \div \mathbf{A}) \mathbf{Z},
$$

where $\mathbf{Z}$ is an arbitrary column vector.

For a diagonal matrix $\mathbf{D}=\operatorname{diag}\left(d_{1}, d_{2}, \ldots, d_{n}\right)$, the M-P inverse of $\mathbf{D}$ is $\mathbf{D} \div=$

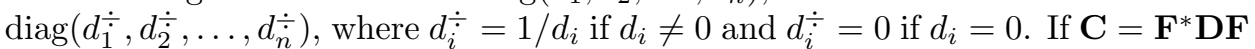
is a circulant, its M-P inverse is given by $\mathbf{C}^{\div}=\mathbf{F}^{*} \mathbf{D}^{\div} \mathbf{F}$.

\section{ACKNOWLEDGEMENT}

The authors wish to thank Professor M. A. Jaswon of the Department of Mathematics, the City University, London, for reading the manuscript and his helpful comments.

\section{REFERENCES}

1. W. H. Chu, Development of a general finite difference approximation for a general domain. Part I: Machine Transformation, J. Comp. Phys. 8 (1971), 392-408.

2. C. W. Mastin and J. F. Thompson, Transformation of three-dimensional regions onto rectangular regions by elliptic systems, Numer. Math. 29 (1978), 397-407. MR 57:14491

3. H. J. Haussling and R. M. Coleman, A method for generation of orthogonal and nearly orthogonal boundary-fitted coordinate systems, J. Comp. Phys. 43 (1981), 373-381. MR 82m:65026

4. C. I. Christov, Orthogonal coordinate meshes with manageable Jacobian, Appl. Math. Comp. 10 (1982), 885-894. CMP 15:03

5. J. F. Thompson, Z. U. A. Warsi, and C. W. Mastin, Boundary-fitted coordinate systems for numerical solution of partial differential equations - a review, J. Comp. Phys. 47 (1982), 1-108. MR 84e:65120

6. P. D. Sparis, A method for generating boundary-orthogonal curvilinear coordinate systems using a biharmonic equation, J. Comp. Phys. 61 (1985), 445-462. MR 87b:65218

7. D. C. Ives, A modern look at conformal mapping including multiply connected regions, AIAA J. 14 (1976), 1006-1011. MR 58:3956

8. O. Hübner, The Newton method for solving the Theodorsen equation, J. Comp. Appl. Math. 14 (1986), 19-30. MR 87c:30012

9. D. I. Meiron, S. A. Orszag, and M. Israeli, Applications of numerical conformal mapping, J. Comput. Phys. 40 (1981), 345-360. MR 83e:30011

10. Z. Nehari, Conformal Mapping, Dover Publications, New York, 1975. MR 51:13206

11. G. T. Symm, An integral equation method in conformal mapping, Numer. Math. 9 (1966), 250-258. MR 34:7056

12. , Numerical mapping of exterior domains, Numer. Math. 10 (1967), 437-445. MR 36:3525

13. M. A. Jaswon and G. T. Symm, Integral Equation Methods in Potential Theory, Academic Press, London, 1971.

14. D. M. Hough and N. Papamichael, The use of splines and singular functions in an integral equation method for conformal mapping, Numer. Math. 37 (1981), 133-147. MR 82e:30012

15. J. P. Berrut, A Fredholm integral equation of the second kind for conformal mapping, J. Comp. Appl. Math. 14 (1986), 99-110. MR 87d:30011 
16. T. Theodorsen, Theory of Wing Sections of Arbitrary Shape, NACA Report 411, 1931.

17. T. Theodorsen and I. E. Garrick, General Potential Theory of Arbitrary Wing Sections, NACA Report 452, 1933.

18. R. Timman, The direct and the inverse problem of aerofoil theory, a method to obtain numerical solutions, Nat. Luchtiv. Labor., Report F.16, Amsterdam, 1951.

19. M. S. Friberg, A new method for the effective determination of conformal maps, Ph.D. Thesis, Univ. of Minnesota, 1951.

20. H. Bergström, An approximation of the analytic function mapping: a given domain inside or outside the unit circle, Mem. Publ. Soc. Sci. Arts Lettr. Hainant, Volume Hors Serie (1958), 193-198. MR 22:8112

21. B. A. Vertgeim, Approximate construction of some conformal mappings (in Russian), Doklady Akad. Nauk SSSR 119 (1958), 12-14. MR 20:5854

22. R. Wegmann, Ein Iterationsverfahren zur komformen Abbildung, Numer. Math. 30 (1978), 453-466. MR 58:11345

23. _ On Fornberg's numerical method for conformal mapping, SIAM J. Numer. Anal. 23 (1986), 1199-1213. MR 87m:30012

24. - An iterative method for the conformal mapping of doubly connected regions, J. Comp. Appl. Math. 14 (1986), 79-98. MR 87e:30008

25. B. Fornberg, A numerical method for conformal mappings, SIAM J. Sci. Stat. Comput. 1 (1980), 386-400. MR 81 m:30009

26. _ A numerical method for conformal mapping of doubly connected regions, SIAM J. Sci. Stat. Comput. 5 (1984), 771-783. MR 86b:30009

27. D. Howe, The application of numerical methods to the conformal transformation of polygonal boundaries, J. Inst. Math. Appl. 12 (1973), 125-136. MR 49:8297

28. V. V. Vecheslavov and V. I. Kokoulin, Determination of the parameters of the conformal mapping of simply connected polygonal regions, U.S.S.R. Comp. Math. and Math. Phys. 13 (1974), 57-65.

29. L. N. Trefethen, Numerical computation of the Schwarz-Christoffel transformation, SIAM J. Sci. Stat. Comput. 1 (1980), 82-102. MR 81g:30012a

30. F. D. Gakhov, Boundary Value Problems, Pergamon Press, Oxford, 1966. MR 33:6311

31. M. H. Gutknecht, Numerical conformal mapping methods based on function conjugation, J. Comp. Appl. Math. 14 (1986), 31-77. MR 87f:30015

32. P. K. Banerjee and R. Butterfield, Boundary Element Methods in Engineering Science, McGraw-Hill, London, 1981. MR 83j:65111

33. P. Henrici, Applied and Computational Complex Analysis, Vol. 3, John Wiley \& Sons, New York, 1986. MR 87h:30002

34. R. Wegmann, An estimate for crowding in conformal mapping to elongated regions, Complex Variables 18 (1992), 193-199. MR 93f:30007

35. T. K. DeLillo, The accuracy of numerical conformal mapping methods: a survey of examples and results, SIAM J. Numer. Anal. 31 (1994), 788-812. MR 95a:30005

36. P. J. Davis, Circulant Matrices, John Wiley \& Sons, New York, 1979. MR 81a:15003

Computervision R\&D, 138-144 London Road, Wheatley, Oxon OX33 1JH, United KingDOM

E-mail address: baoli@cvedg.cv.com

Department of Mechanical Engineering, University of Southampton, Highfield, Southampton SO17 1BJ, United Kingdom

E-mail address: ss@soton.ac.uk 